\title{
Comparison of transanal endoscopic microsurgery with or without neoadjuvant therapy and standard total mesorectal excision in the treatment of clinical T2 low rectal cancer: a meta-analysis
}

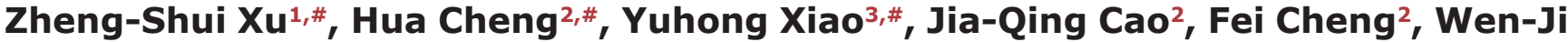 \\ $\mathrm{Xu}^{2}$, Jia-Qi Ying ${ }^{2}$, Jun Luo ${ }^{4, *}$ and Wei $\mathrm{Xu}^{2, *}$ \\ ${ }^{1}$ Department of General Surgery, Xi'an N0.4 Hospital, 710000 Xi'an, Shanxi, China \\ ${ }^{2}$ Department of General Surgery, The Second Affiliated Hospital of Nanchang University, 330006 Nanchang, Jiangxi, China \\ ${ }^{3}$ The Second Clinical Medical College, Nanchang University, 330006 Nanchang, Jiangxi, China \\ ${ }^{4}$ Department of Rehabilitation Medicine, The Second Affiliated Hospital of Nanchang University, 330006 Nanchang, Jiangxi, \\ China \\ \#These authors contributed equally to this study and share first authorship \\ *These authors contributed equally to this study and share corresponding authorship \\ Correspondence to: Wei Xu, email: xu_wei111@126.com \\ Jun Luo, email: luojun1786@163.com
}

Keywords: rectal cancer, transanal endoscopic microsurgery, neoadjuvant therapy, total mesorectal excision, meta-analysis Received: December 10, $2016 \quad$ Accepted: September 18, $2017 \quad$ Published: October 26, 2017

Copyright: $\mathrm{Xu}$ et al. This is an open-access article distributed under the terms of the Creative Commons Attribution License 3.0 (CC BY 3.0), which permits unrestricted use, distribution, and reproduction in any medium, provided the original author and source are credited.

\section{ABSTRACT}

Some clinical trials demonstrated local resection for clinical T1 rectal cancer was safe and effective. But for clinical $\mathrm{T} 2$ rectal cancer, the results were controversial. Neoadjuvant therapy (NT) is proven to reduce the opportunity of advanced rectal cancer recurrence in various researches. The objective of this Meta-Analysis was to evaluate the oncological outcomes of transanal endoscopic microsurgery (TEM) with or without NT comparing with conventional total mesorectal excision (TME) for the treatment of clinical T2 rectal cancer.To search for the relevant studies, an electronic search was done from the databases of Pubmed, Embase, and the Cochrane Library in this meta-analysis. We compared the effectiveness of transanal endoscopic microsurgery with or without NT and standard total mesorectal excision in the treatment of T2 Rectal Cancer. 1RCT and 3nRCTs including 121 TEM patients (TEM + NT: 59, TEM: 62) and 174 TME patients with T2 rectal cancer were retrieved. Compared with TME, there were no significant differences in the outcomes of local recurrence, overall recurrence, overall survival between TEM + NT group. However in compassion with TME, TEM without NT was associated with an increased local recurrence, overall recurrence, and a shorter overall survival, with individual ORs being 3.04 (95\% $\mathrm{Cl}$ : $\left.1.17-7.90 ; \mathrm{I}^{2}=0 \%\right), 5.67\left(95 \% \mathrm{Cl}: 1.58-20.38 ; \mathrm{I}^{2}=0 \%\right)$ and $0.12(95 \% \mathrm{Cl}: 0.02-$ $0.65 ; I^{2}=0 \%$ ), respectively. Compared with TME, TEM after NT may be a feasible and safe organ preservative approach for patients with clinical T2 low rectal cancer. But for those without NT, TEM always seem be associated with worse oncological outcomes.

\section{INTRODUCTION}

Rectal cancer remains one of the most common malignancies in the world, especially in the western countries and some developing countries [1,2]. In the past twenty years, the five-year overall survival rate of rectal cancer was greatly increased, as a result of early detection and treatment was spread [1]. By 2009, early-stage rectal 
cancers (T1-T2) were nearly one quarter of all newly diagnosed ones in the UK [3]. Standard total mesorectal excision (TME) surgery, as another reason for the increased 5 -year survival rate of rectal cancer, is an oncologically effective treatment for early-stage rectal cancers with extremely low rate of local and distant recurrence [4]. Unfortunately, TME-related complications are substantial, which include sexual and urinary dysfunction, troubling defecatory problems, decreased quality of life or a permanent stoma [5-7]. Some organ-preserving treatments were evaluated as the alternative approach of standard TME for the early-stage rectal cancers.

In 1983, Transanal endoscopic microsurgery (TEM) was firstly proposed for the treatment of large adenomas and clinical T1 rectal cancer [8]. But a major problem of TEM is the possibility of lymph node metastasis, which is the source for the local and remote recurrence, though its rate is very low in the early stage rectal cancer $[9,10]$. The current evidence describes that TEM has gained general acceptance for the treatment of selected clinical T1 rectal cancer patients, because lots of reports demonstrate the same 5-year survival rate, similar recurrence rate and better functional results are followed with compared with standard TME resection [11-14]. But for clinical T2 rectal cancer, with higher rate of lymph node metastasis, the oncological outcomes and long-term survival are controversial. Neoadjuvant therapy (NT), compared with postoperative therapy, is proven to reduce the opportunity of advanced rectal cancer recurrence in a number of researches [15-17]. After all, NT maybe a suitable strategy for the probable lymph node metastasis leaved by TEM approach for the treatment of clinical $\mathrm{T} 2$ rectal cancer.

In the past 20 years, more and more practice experience were reported that TEM (with or without NT) maybe feasible and safety for treating clinical T2 rectal cancer [18-21]. Within these articles, some reports were compared the short-time outcomes and long-term survival between TEM (with or without NT) and standard TME, and there were many meta-analyses which assessed the effectiveness of TEM for stage (including T1 and T2) rectal cancer [18-21]. However, those results cannot be applied to T2 rectal cancer, because TEM as a surgical strategy for T1 rectal cancer has a consensus statement by NCCN Clinical Practice Guidelines in Rectal Cancer [22]. Those meta-analyses could not demonstrate the real pooled results in $\mathrm{T} 2$ rectal cancer with a too heavy weight of $\mathrm{T} 1$ rectal cancer. So we performed this meta-analysis to compare the effectiveness of transanal endoscopic microsurgery and total mesorectal excision in the treatment of T2 Rectal Cancer.

\section{MATERIALS AND METHODS}

\section{Literature search}

An electronic search was done from the databases of Pubmed, Embase, and the Cochrane Library to search for the relevant studies which were published from inception of these databases to march 19, 2016. No restrictions were entered for the search. The search strategy was performed using the following terms: rectal cancer, total mesorectal excision, transanal endoscopic microsurgery; Details of literature search were shown in appendix 1. Two investigators performed repeatedly the search strategy until no further relevant studies were retrieved; then two investigators assessed independently all the article using pre-designed eligibility forms; References of the above eligibility studies were also checked manually to add other potential eligibility studies. Any discrepancies were resolved by consensus. The scheme for this process was shown in Figure 1.

\section{Inclusion criteria and exclusion criteria}

Eligibled studies should met the following criteria: (a) the diagnosis of T2 rectal cancer should be included pathological examination, clinical evaluation, colonoscopy and one or more imaging examination; (b) published studies comparing TEM and TME; (c) the sufficient data of our interesting. The excluded criteria was shown as follow: (a) the aim of trails were not T2 rectal cancer; (b) duplicate data or repeat analysis; (d) lack of sufficient data which we are interesting or cannot be calculated from the article data. Table 2 Pre-therapy protocol and follow-up adopted in included trials.

\section{Data collection and quality assessment}

The data of our interest were extracted independently; the results were compared and checked by another co-author. From each study the following information were collected: first author, year of publication, country of origin, study design, age, gender, total number of cases, with or without NT, the outcome (local recurrence, overall recurrence, overall survival, postoperative complications) and followup. The quality assessment of non-randomized controlled clinical trials (nRCTs) or randomized controlled trials(RCTs) was done using the Newcastle-Ottawa Scale (range 0-9) [23] or a Jadad score (range 0-5) [24], respectively.

\section{Statistical analysis}

We conducted this meta-analysis according to the recommendations of the Preferred Reporting Items for Systematic Reviews and Meta-Analysis (PRISMA) Statement. [25] Heterogeneity among the included studies was evaluated using the $\mathrm{I}^{2}$ values. An $\mathrm{I}^{2}$ value of is equal or lesser than $50 \%$ showed no significant heterogeneity, and the fixed-effects model was used. Otherwise, a randomeffects model was reported, and it represented substantial levels of heterogeneity. Because of all pooled outcomes which were less than 10 included trials, publication bias would not be evaluated. All data were analyzed 
using the Review Manager Version 5.2 (The Cochrane Collaboration, Oxford).

\section{RESULTS}

\section{Study characteristics}

The initial database search identified 490 publications. Through removing the duplicates by electron and handed-scan, 365 publications were left. After reviewing the title and abstract of all the 365 publications, we identified 14 potential eligible studies. Finally 6 studies was left, however 3 studies [26-28] reported the similar outcomes at different follow-up point. Eventually, 1 RCT and 3nRCTs including 121 TEM patients (TEM + NT: 59, TEM: 62) and 174 TME patients with T2 rectal cancer were retrieved, which published between 2003 and 2013 [28-31]. In the Allaix's study, two cases (18\%) of all patients with local tumor progression in neoadjuvant treatment group - both patients underwent open surgery were observed, in this condition we supposed that two cases had the worst results that both cases were classified as "event group" [30]. The characteristics of all including studies were summarized in Table 1, and the pre-therapy protocol and follow-up adopted in included trials were presented in Table 2.

\section{Oncological outcomes}

\section{TEM + NT vs TME}

Compared with TME, there were no significant differences in the outcomes of local recurrence (Figure 2A), overall recurrence (Figure 3A), overall survival (Figure 4A) between TEM + NT group.

\section{TEM only vs TME}

Compared with TME, TEM only was associated with a increased local recurrence (Figure 2B), overall recurrence (Figure 3B), and a shorter overall survival (Figure 4B), with individual ORs being $3.04(95 \% \mathrm{Cl}$ : $\left.1.17-7.90 ; \mathrm{I}^{2}=0 \%\right), 5.67\left(95 \% \mathrm{Cl}: 1.58-20.38 ; \mathrm{I}^{2}=0 \%\right)$ and $0.12\left(95 \% \mathrm{Cl}: 0.02-0.65 ; \mathrm{I}^{2}=0 \%\right)$, respectively.

\section{DISCUSSION}

Low anterior resection (LAR) or abdominoperineal resection (APR) with total mesorectal excision is widespread accepted as the gold standard procedure in early stage rectal cancer, with the same oncological results between the open and the laparoscopic approach [32-34]. Laparoscopic surgery has proven to have better shortterm clinical outcomes than open approach, which is also burdened by high rate of morbidity and mortality. The rate

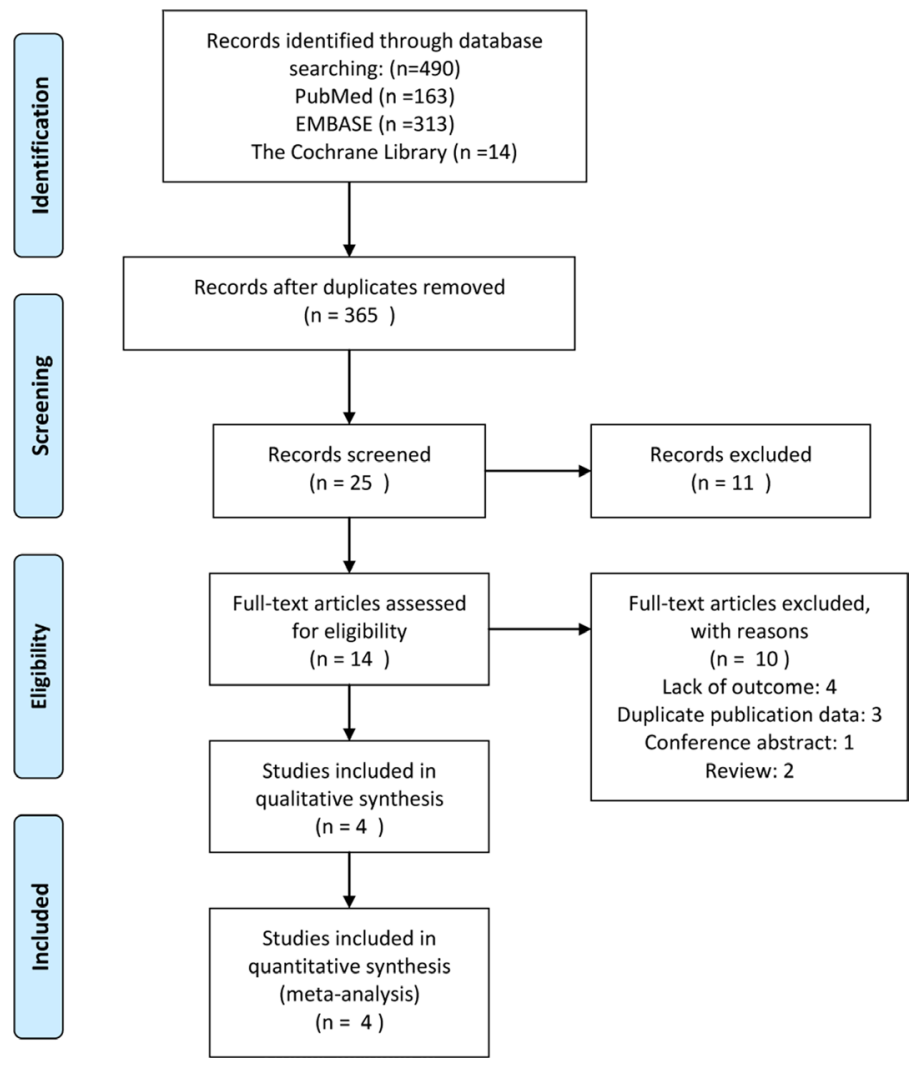

Figure 1: Diagram of study selection process. 
Table 1: Characteristics of included studies in current meta-analysis

\begin{tabular}{|c|c|c|c|c|c|c|c|c|c|}
\hline Author & Year & Country & study design/quality ${ }^{*}$ & Age(years) & $\begin{array}{c}\text { Gender } \\
(\mathrm{M} / \mathrm{F})\end{array}$ & $\begin{array}{c}\text { Neoadjuvant } \\
\text { therapy }\end{array}$ & $\begin{array}{c}\text { Follow-up } \\
\text { (months) }\end{array}$ & $\begin{array}{l}\text { TNM } \\
\text { stage }\end{array}$ & $\begin{array}{c}\text { Histological } \\
\text { grade }\end{array}$ \\
\hline Allaix & 2012 & Italy & $\begin{array}{l}\text { Retrospective analysis } \\
\text { of Prospective } \\
\text { database } / 6\end{array}$ & $\begin{array}{l}72(38-91) \\
65(34-90)\end{array}$ & $\begin{array}{l}16: 25 \\
16: 19\end{array}$ & $\begin{array}{l}\text { A part of } \\
\text { patients }\end{array}$ & $70(36-140)$ & T2N0M0 & NA \\
\hline Chen & 2013 & China & Prospective Studies/6 & $\begin{array}{l}68.8 \pm 5.3 \\
66.2 \pm 7.7\end{array}$ & $\begin{array}{l}14: 16 \\
17: 13\end{array}$ & No patient & $\begin{array}{l}18 \pm 2.6 \\
17.5 \pm 2.2\end{array}$ & T2N0M0 & G1-2 \\
\hline Lee & 2003 & $\begin{array}{l}\text { South } \\
\text { Korea }\end{array}$ & Retrospective/8 & $\begin{array}{l}61.1 \pm 11.2 \\
57.7 \pm 11.8\end{array}$ & $\begin{array}{l}37: 37 \\
50: 50\end{array}$ & No patient & $\begin{array}{l}31 \pm 17.2 \\
34.6 \pm 19.4\end{array}$ & T2N0M0 & G1-2 \\
\hline Lezoche & 2012 & Italy & $\begin{array}{l}\text { Randomized, } \\
\text { controlled trial/4 }\end{array}$ & $\begin{array}{l}66(58-70) \\
66(60-69)\end{array}$ & $\begin{array}{l}30: 20 \\
34: 16\end{array}$ & All patients & $\begin{array}{l}115.2 \\
(102-133.2) \\
115.2 \\
(88.8-142.8)\end{array}$ & T2N0M0 & G1-2 \\
\hline
\end{tabular}

"Quality assessment was done using the Newcastle-Ottawa Scale (range 0-9) for non-randomised studies and using a Jadad score (range 0-5) for randomized, controlled trials. NA: No applicable.

of mortality was $2 \%$ to $6 \%$ after radical rectal surgery, and this rate could be higher in elderly patients $[35,36]$; the major complications were urinary and sexual dysfunction, long-term functional bowel disturbance, and anastomotic leakage [37-39]. With temporary or permanent stoma, patients may suffer significant psychological problems, which will lead to worsening of the quality of life
(QoL) [40, 41]. For early stage rectal cancer, oncological outcomes should be the primary aim, and secondly preserving function and QoL should pay more attention on.

The poor QoL and high rate of morbidity drive surgeons to reconsider the role of local therapy for early stage rectal cancer, which could preserve integrity of anal sphincter and pelvic autonomic nerve to maintain
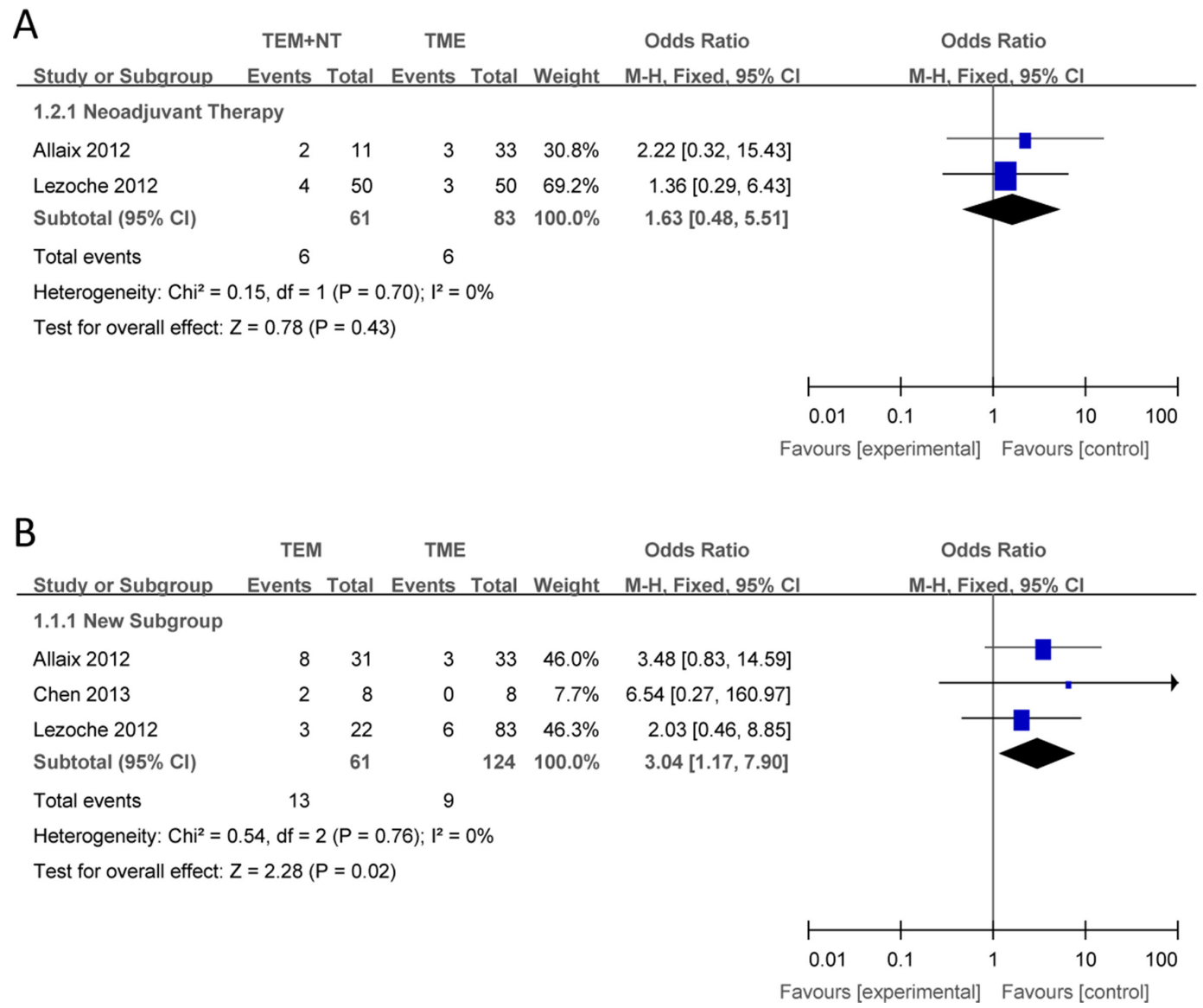

Figure 2: Forest plots of local recurrence, TEM + NT VS TME (A), TEM only VS TME (B). TEM = transanal endoscopic microsurgery, $\mathrm{TME}=$ total mesorectal excision, $\mathrm{NT}=$ neoadjuvant therapy, $\mathrm{CI}=$ confidence intervals, $\mathrm{MH}=$ Mantel-Haenszel. 
Table 2: Pre-therapy protocol and follow-up adopted in included trials

Trial TEM group

\section{TME group}

Neoadjuvant therapy: radiotherapy of 45 Gy for No neoadjuvant treatment. Because of 2 patient lost 6-8 weeks for all TEM patients. Two cases (18\%) to follow-up, That 33 patients undergone TME were of all neoadjuvant treatment with local tumor inclued in our study. progression (both patients underwent open surgery)

Allaix 2012 were observed, in this condition we supposed that two cases had the worst results that both cases were classified as "event group". Because of one patient lost to follow-up 11 patients, 32 patients undergone TEM+NT, TEM only, respectively.

Chen 2013 No neoadjuvant treatment. 8 patients undergone TEM. No patient lost to follow-up.

No neoadjuvant treatment. 8 patientsundergone TME. No patient lost to follow-up.

No neoadjuvant treatment. 83 patientsundergone TME. No patient lost to follow-up. TEM. No patient lost to follow-up.

Neoadjuvant therapy: long course 3D four-field chemoradiotherapy in prone position, with bladder prep and use of IV contrast Total dose 5,040

Lezoche 2012 cGy in 28 fractions over 5 weeks with infusion of 5-fluorouracil $200 \mathrm{mg} / \mathrm{m}^{2}$ per day during radiotherapy. 50 patients undergone TME. No patient lost to follow-up.
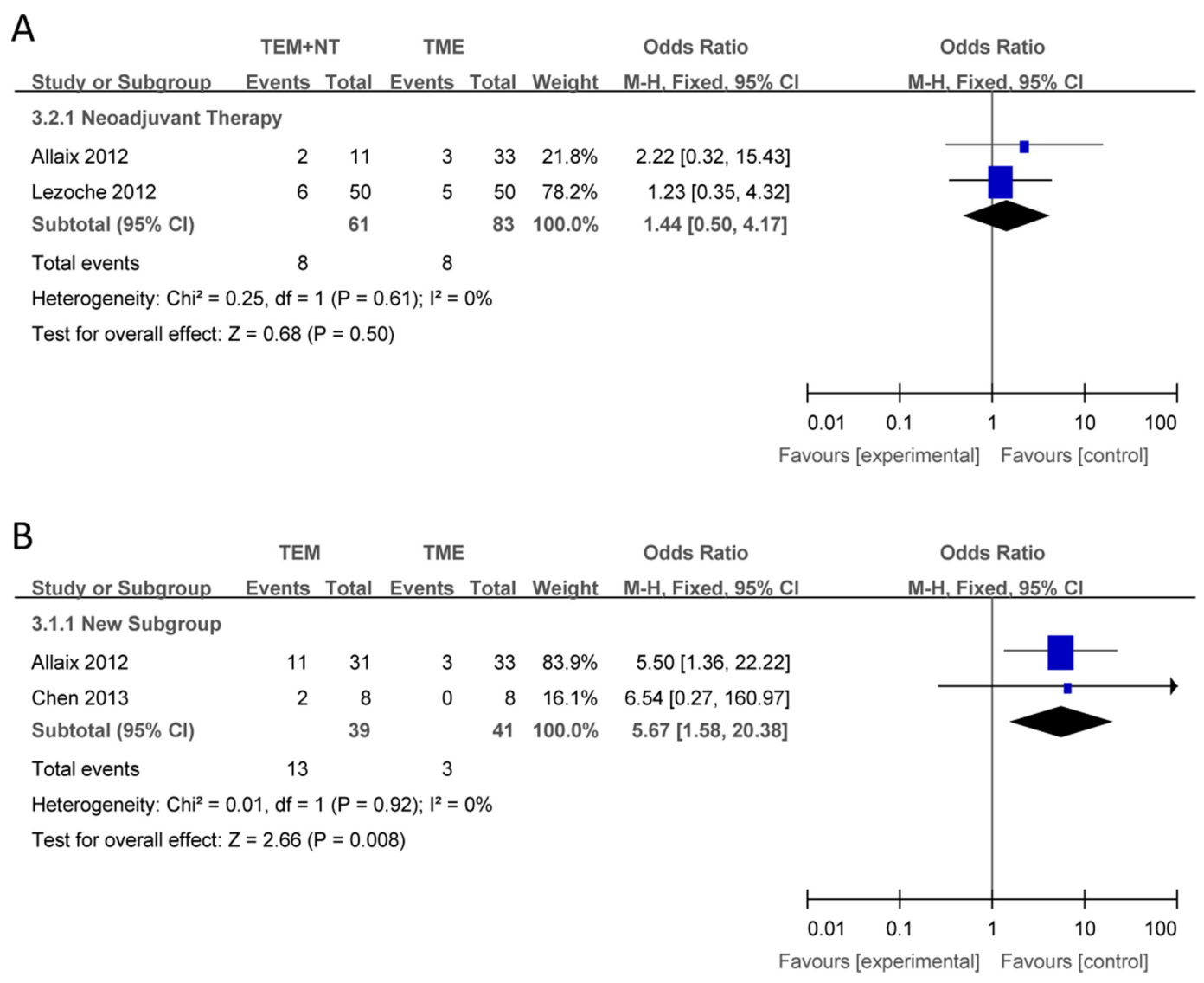

Figure 3: Forest plots of local recurrence, TEM + NT VS TME (A), TEM only VS TME (B). TEM = transanal endoscopic microsurgery, $\mathrm{TME}=$ total mesorectal excision, $\mathrm{NT}=$ neoadjuvant therapy, $\mathrm{CI}=$ confidence intervals, $\mathrm{MH}=$ Mantel-Haenszel. 
the best QoL. TEM, as a kind of local excision, was firstly introduced for the treatment of large rectal polyps and early rectal cancer in 1983 [42]. As compared with traditional local resection approach, TEM achieves a better vision of operation area, which allows a very accurate and full-thickness excision of the tumor and mesorectum down to the 'holy plane' with an about $1 \mathrm{~cm}$ surrounding tumor-free margin. A R0 resection margin of the specimen is significant importance for the rate of local recurrence.

Currently, TEM for rectal magligant tumor is just accepted for clinical T1 adenocarcinomas with favorable prognostic characteristics. In these patients, the rate of local recurrence and overall survival is similar compared with conventional radical TME surgery [43-45]. But for clinical T2 rectal cancer, with about 20\% lymph node metastasis rate, the oncological outcomes and long-term survival of TEM are controversial [46]. Following the development of NT in rectal cancer, it improves the local control of rectal cancer. NT can lead to a complete clinical response (CCR) in 10\%-30\% of rectal patient [47]. This result brings about a new "wait and see" strategy. However smith et al [48] found that the residual mucosal abnormalities which were less than $3 \mathrm{~cm}$ had significantly association with ypT0-1 after neoadjuvant therapy for rectal cancer. And a CCR did not mean a complete pathological response $(\mathrm{CPR})$ in some cases. So TEM +
NT could be commended as an ontologically adequate treatment. But there are a very limited number of clinical trials comparing TEM with or without neoadjuvant therapy and radical surgery for clinical $\mathrm{T} 2$ rectal cancer. Our present research analyzed 4 studies, including 1 RCT $+3 \mathrm{nRCT}$, which are focused on different short-term and long-term outcomes. Our primary endpoint is the local recurrence, overall recurrence and overall survival, which are paramount points of oncological outcomes. As the results described in our meta-analysis, for patients with clinical T2 low rectal cancer after NT, the local recurrence, overall recurrence and overall survival is similar with TME surgery; unfortunately, for clinical T2 patients without NT, the results of local recurrence rate, overall recurrence and overall survival are significantly worse than radical surgery. NT is the key point for this favorable outcome, because of it could reduce the local infiltration depth of cancer and the possibility of mesorectal lymph nodes metastasis [15-17].

Besides these, we must emphasize the advantage of TEM in terms of QoL. Although a nerve sparing technique is applied to laparoscopic TME, the anterior resection syndrome, including urinary dysfunctions, sexual, and variable defecation, still occurs with the rate of $50 \%$ $90 \%$ [49-52]. Obviously, TEM thoroughly win in this term $[53,54]$. Moreover two studies reported that there
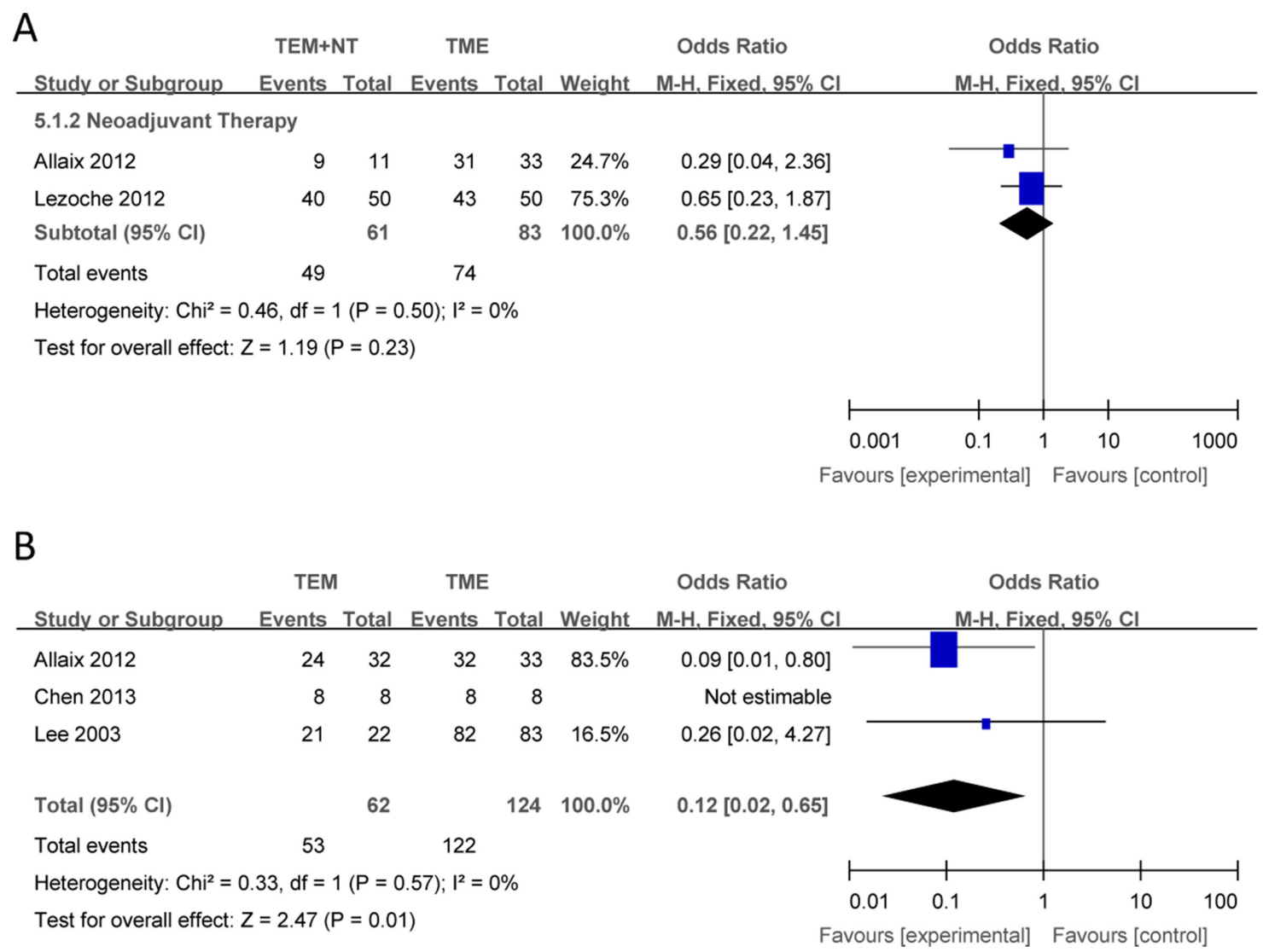

Figure 4: Forest plots of overall survival, TEM +NT VS TME (A), TEM only VS TME (B). TEM = transanal endoscopic microsurgery, $\mathrm{TME}=$ total mesorectal excision, $\mathrm{NT}=$ neoadjuvant therapy, $\mathrm{CI}=$ confidence intervals, $\mathrm{MH}=$ Mantel-Haenszel. 
are no significant differences in QoL between TEM and TEM after NT for locally advanced rectal cancer [54-55]. Many authors recommended that a temporary or definitive stoma should be applied to low anterior resection, it goes without saying that it would increase the prevalence of depression and worse the quality of life and, especially in some cultural contexts $[41,56]$.

Many studies proved that TEM with or without NT have a better short-term outcomes compared with TME, including blood loss, transfusions, operating time, need for analgesia and hospital stay [27, 43, 57]. Compared with TME, there are lower life-threatening complication occurred after TEM with or without NT, this advantage would be obvious in the patient with American Society of Anesthesiologists' (ASA) class III or IV [27, 57].

However, every nutshell has a concave and convex side. Firstly, chemoradiotherapy-related toxicity is a concern in the patient with early stage rectal cancer who should not have to receive neoadjuvant therapy before TME, mortality incident is the most fearful; although some studies reported that mortality incident on chemoradiotherapy is less than $1 \%[57,58]$. Secondly, treatment-related toxicities after TEM+NT can be neglected, including anorectal pain, proctitis, diarrhoea, suture dehiscence and persistent confusion et al. [58, 59]. Thirdly, some patients who treated with TEM + NT may still need TME; these patients were overtreatment with additional morbidity of NT and TEM [58]. Because of the fibrotic scar in the rectal wall and the local inflammation after TEM, the pelvic dissection and a low colorectal or a coloanal anastomosis would be more difficult [43]. Beiside these, NT would increase the rate of anastomositis, marginal ulcer, anastomotic fistula et al. [43, 57-60].

What are the future problems in the development of this procedure? It is not completely accurate for the preoperative staging of the tumors, compared with the definitive histological stage. Even though, EUS was considered as the most accurate preoperative diagnostic tool for the tumor invasion of the rectal wall and high quality of MRI for the lymph node condition. What are next steps in the development of this procedure? The next study should be refined. Firstly, it is important that ypT2 rectal cancer should be divide into a group, because it is the most ambiguous whether TEM + NT is applicable to ypT2 rectal cancer. Secondly, it should be clear whether the diameter, the different pathologic types and grades of T2 rectal cancer would make different outcomes. Finally, whether and how would TEM + NT be applicant to clinical T3 rectal cancer?

There were some limitations to our study. First, there are an insufficient number of research studies in comparsion of transanal endoscopic microsurgery with or without NT and standard total mesorectalexcision in thetreatment of clinical T2 low rectal cancer, and only $1 \mathrm{RCT}$ and $3 \mathrm{nRCTs}$ with nearly 300 patients were included in this metaanalysis. Second, because of insufficient data of short-term outcomes, they cannot be analyzed in this meta-analysis. Moreover, these 4 including studies were published in English, so publication bias cannot be excluded. Thus, we reckoned that more attention should been paid to TEM with NT in the treatment of clinical T2 low rectal cancer, and this is one reason why we written this article.

\section{CONCLUSIONS}

Compared with TME, TEM may be a feasible and safe organ preservative approach for patients with clinical T2 low rectal cancer after NT. But for those without NT, TEM always seem be associated with worse oncological outcomes. Anyhow it is good news for the patients with T2 low rectal cancer refusing abdominal surgery or unfit for TME because of severe comorbidities.

\section{CONFLICTS OF INTEREST}

None.

\section{GRANT SUPPORT}

This study was supported by Natural Fund Project of Jiangxi Province [20151BAB205086, Zeng Fei, PI].

\section{REFERENCES}

1. Siegel RL, Miller KD, Jemal A. Cancer statistics, 2016. CA Cancer J Clin. 2016; 66:7-30.

2. Tsoi KKF, Hirai HW, Chan FCH, Griffiths S, Sung JJY. Predicted Increases in Incidence of Colorectal Cancer in Developed and Developing Regions, in Association with Ageing Populations. Clinical Gastroenterology and Hepatology. 2017; 15:892-900.

3. Cancer Research UK. Bowel Cancer Statistics. http://www. cancerresearchuk.org/cancer-info/cancerstats/types/bowel/ incidence/uk-bowel-cancer-incidence-statistics [accessed 29 August 2014].

4. Primrose JN, Perera R, Gray A, Rose P, Fuller A, Corkhill A, George S, Mant D; FACS Trial Investigators. FACS Trial Investigators. Effect of 3 to 5 years of scheduled CEA and CT follow-up to detect recurrence of colorectal cancer: the FACS randomized clinical trial. JAMA. 2014; 311:263-270.

5. Paun BC, Cassie S, MacLean AR, Dixon E, Buie WD. Postoperative complications following surgery for rectal cancer. Ann Surg. 2010; 251:807-818.

6. Lange MM, van de Velde CJ. Urinary and sexual dysfunction after rectal cancer treatment. NatRev Urol. 2011; 8:51-57.

7. Floodeen H, Lindgren R, Hallbook O, Matthiessen P. Evaluation of long-term anorectal function after low anterior resection: a 5-year follow-up of a randomized multicenter trial. Dis Colon Rectum. 2014; 57:1162-68. 
8. Buess G, Theiss R, Hutterer F, Pichlmaier H, Pelz C, Holfeld T, Said S, Isselhard W. Transanal endoscopic surgery of the rectum-testing a new method in animal experiments. Leber Magen Darm. 1983; 13:73-77.

9. Mellgren A, Sirivongs P, Rothenberger DA, Madoff RD, GarciaAguilar J. Is local excision adequate therapy for early rectal cancer? Dis Colon Rectum. 2000; 43:1064-1074.

10. Minsky BD, Rich T, Recht A, Harvey W, Mies C. Selection criteria for local excision with or without adjuvant radiation therapy for rectal cancer. Cancer. 1989; 63:1421-1429.

11. Heintz A, Morschel M, Junginger T. Comparison of results after transanal endoscopic microsurgery and radical resection for T1 carcinoma of the rectum. Surg Endosc. 1998; 12:1145-1148.

12. Palma P, Freudenberg S, Samel S, Post S. Transanal endoscopic microsurgery: indications and results after 100 cases. Colorectal Dis. 2004; 6:350-355.

13. Sengupta S, Tjandra JJ. Local excision of rectal cancer: what is the evidence? Dis Colon Rectum. 2001; 44:1345-1361.

14. Varma MG, Rogers SJ, Schrock TR, Welton ML. Local excision of rectal carcinoma. Arch Surg. 1999; 134:863-867, discussion 867-868.

15. Sebag-Montefiore D, Stephens RJ, Steele R, Monson J, Grieve R, Khanna S, Quirke P, Couture J, de Metz C, Myint AS, Bessell E, Griffiths G, Thompson LC, et al. Preoperative radiotherapy versus selective postoperative chemoradiotherapy in patients with rectal cancer (MRC CR07 and NCIC-CTG C016): a multicentre, randomised trial. Lancet. 2009; 373:811-820.

16. Peeters KC, Marijnen CA, Nagtegaal ID, Kranenbarg EK, Putter H, Wiggers T, Rutten H, Pahlman L, Glimelius B, Leer JW, van de Velde CJ; Dutch Colorectal Cancer Group. The TME trial after a median follow-up of 6 years: increased local control but no survival beneft in irradiated patients with resectable rectal carcinoma. Ann Surg. 2007; 246:693-701.

17. Sauer R, Liersch T, Merkel S, Fietkau R, Hohenberger W, Hess C, Becker H, Raab HR, Villanueva MT, Witzigmann H, Wittekind C, Beissbarth T, Rödel C. Preoperative versus postoperative chemoradiotherapy for locally advanced rectal cancer: results of the German CAO/ARO/AIO-94 randomized phase III trial after a median follow-up of 11 years. J Clin Oncol. 2012; 30:1926-1933.

18. Sajid MS, Farag S, Leung P, Sains P, Miles WF, Baig MK. Systematic review and meta-analysis of published trials comparing the effectiveness of transanal endoscopic microsurgery and radical resection in the management of early rectal cancer. Colorectal disease. 2014; 16:2-14.

19. Chakravorty V, Chamberlain R, Ghlayiae N. Transanal endoscopic microsurgery (TEM) versus total mesorectal excision (TME) radical resection for $\mathrm{T} 1$ or $\mathrm{T} 2$ rectal cancer - A metaanalysis. Diseases of the colon and rectum. 2015; 58:e170.
20. Carrara A, Mangiola D, Zappalà O, Silvestri M, Del Balzo R, De Pretis G, Tirone G. Local excision as single treatment for early stages of rectal cancer: Indications and causes of local failure. Systematic literature review and metaanalysis. Techniques in coloproctology. 2011; 15:235.

21. Chiniah M, Ganganah O, Cheng Y, Sah SK. Transanal endoscopic microsurgery is an oncologically safe alternative to total mesorectal excision for stage I rectal cancer: results of a meta-analysis of randomized controlled trials. International journal of colorectal disease. 2016; 31:15011504.

22. NCCN Clinical Practice Guidelines in Oncology [2015]: Rectal Cancer. 2015-2. Fort Washington, PA: National Comprehensive Cancer Network; 2009.

23. Jadad AR, Moore RA, Carroll D, Jenkinson C, Reynolds DJ, Gavaghan DJ, McQuay HJ. Assessing the quality of reports of randomized clinical trials: is blinding necessary? Control Clin Trials. 1996; 17:1-12.

24. Stang A. Critical evaluation of the Newcastle-Ottawa scale for the assessment of the quality of nonrandomized studies in meta-analyses. Eur J Epidemiol. 2010; 25:603-605.

25. Liberati A, Altman DG, Tetzlaff J, Mulrow C, Gøtzsche PC, Ioannidis JP, Clarke M, Devereaux PJ, Kleijnen J, Moher D. The PRISMA statement for reporting systematic reviews and meta-analyses of studies that evaluate health care interventions: explanation and elaboration. PLoS Med. 2009; 6:e1000100.

26. Lezoche E, Guerrieri M, Paganini AM, D'Ambrosio G, Baldarelli M, Lezoche G, Feliciotti F, De Sanctis A. Transanal endoscopic versus total mesorectal laparoscopic resections of T2-N0 low rectal cancers after neoadjuvant treatment: a prospective randomized trial with a 3-years minimum follow-up period. Surgical endoscopy. 2005; 19:751-756.

27. Lezoche G, Baldarelli M, Guerrieri M, Mario N, Paganini AM, Sanctis A, Bartolacci S, Lezoche E. A prospective randomized study with a 5-year minimum follow-up evaluation of transanal endoscopic microsurgery versus laparoscopic total mesorectal excision after neoadjuvant therapy. Surgical endoscopy. 2008; 22:352-8. https://doi. org/10.1007/s00464-007-9596-y.

28. Lezoche E, Baldarelli M, Lezoche G, Paganini AM, Gesuita $\mathrm{R}$, Guerrieri M. Randomized clinical trial of endoluminal locoregional resection versus laparoscopic total mesorectal excision for T2 rectal cancer after neoadjuvant therapy. British Journal of Surgery. 2012; 99:1211-1218.

29. Lee W, Lee D, Choi S, Chun H. Transanal endoscopic microsurgery and radical surgery for $\mathrm{T} 1$ and $\mathrm{T} 2$ rectal cancer. Surgical endoscopy. 2003; 17:1283-1287.

30. Allaix ME, Arezzo A, Giraudo G, Morino M. Transanal endoscopic microsurgery vs. laparoscopic total mesorectal excision for T2N0 rectal cancer. Journal of gastrointestinal surgery. 2012; 16:2280-2287. 
31. Chen YY, Liu ZH, Zhu K, Shi PD, Yin L. Transanal endoscopic microsurgery versus laparoscopic lower anterior resection for the treatment of T1-2 rectal cancers. Hepatogastroenterology. 2013; 60:727-732.

32. Heald RJ, Ryall RD. Recurrence and survival after total mesorectal excision for rectal cancer. Lancet. 1986; $1: 1479-1482$.

33. Guillou PJ, Quirke P, Thorpe H, Walker J, Jayne DG, Smith AM, Heath RM, Brown JM, MRC CLASICC trial group. Shortterm endpoints of conventional versus laparoscopicassisted surgery in patients with colorectal cancer (MRC CLASICC trial): multicentre, randomised controlled trial. Lancet. 2005; 365:1718-1726.

34. Braga M, Frasson M, Vignali A, Zuliani W, Capretti G, Di Carlo V. Laparoscopic resection in rectal cancer patients: outcome and cost-benefit analysis. Dis Colon Rectum. 2007; 50:464-471.

35. Gooiker GA, Dekker JW, Bastiaannet E, van der Geest LG, Merkus JW, van de Velde CJ, Tollenaar RA, Liefers GJ. Risk factors for excess mortality in the first year after curative surgery for colorectal cancer. Ann Surg Oncol. 2012; 19:2428-2434.

36. van der Sijp MP, Bastiaannet E, Mesker WE, van der Geest LG, Breugom AJ, Steup WH, Marinelli AW, Tseng LN, Tollenaar RA, van de Velde CJ, Dekker JW. Differences between colon and rectal cancer in complications, shortterm survival and recurrences. Int J Colorectal Dis. 2016; 31:1683-1691.

37. Jayne DG, Brown JM, Thorpe H, Walker J, Quirke P, Guillou PJ. Bladder and sexual function following resection for rectal cancer in a randomized clinical trial of laparoscopic versus open technique. Br J Surg. 2005; 92:1124-1132.

38. Quah HM, Jayne DG, Eu KW, Seow-Choen F. Bladder and sexual dysfunction following laparoscopically assisted and conventional open mesorectal resection for cancer. Br J Surg. 2002; 89:1551-1556.

39. Havenga K, Enker WE, McDermott K, Cohen AM, Minsky $\mathrm{BD}$, Guillem J. Male and female sexual and urinary function after total mesorectal excision with autonomic nerve preservation for carcinoma of the rectum. J Am Coll Surg. 1996; 182:495-502.

40. Camilleri-Brennan J, Steele RJ. Objective assessment of morbidity and quality of life after surgery for low rectal cancer. Colorectal Dis. 2002; 4:61-66.

41. Grumann MM, Noack EM, Hoffmann IA, Schlag PM. Comparison of quality of life in patients undergoing abdominoperineal extirpation or anterior resection for rectal cancer. Ann Surg. 2001; 233:149-156.

42. Buess G, Theiss R, Hutterer F, Pichlmaier H, Pelz C, Holfeld T, Said S, Isselhard W. Transanal endoscopic surgery of the rectum-testing a new method in animal experiments. Leber Magen Darm. 1983; 13:73-77.
43. Allaix ME, Arezzo A, Morino M. Transanal endoscopic microsurgery for rectal cancer: T1 and beyond? An evidence-based review. Surg Endosc. 2016; 30:4841-4852. https://doi.org/10.1007/s00464-016-4818-9.

44. Winde G, Nottberg Keller HR, Schmid KW, Bunte H. Surgical cure for early rectal carcinomas (T1): transanal endoscopic microsurgery vs anterior resection. Dis Colon Rectum. 1996; 39:969-976.

45. Recsky MA, Raval M, Phang PT, Karimuddin A, Brown CJ. Comparing outcomes of TEM versus TME for treatment of stage T1 rectal cancer. Colorectal Disease. 2014; 16:174.

46. Sengupta S, Tjandra JJ. Local excision of rectal cancer: what is the evidence? Dis Colon Rectum. 2001; 44:1345-1361.

47. Smith FM, Waldron D, Winter DC. Rectum-conserving surgery in the era of chemoradiotherapy. Br J Surg. 2010; 97:1752-1764.

48. Smith FM, Chang KH, Sheahan K, Hyland J, O'Connell PR, Winter DC. The surgical significance of residual mucosal abnormalities in rectal cancer following neoadjuvant chemoradiotherapy. Br J Surg. 2012; 99:993-1001.

49. Kuzu MA, Topçu O, Uçar K, Ulukent S, Unal E, Erverdi N, Elhan A, Demirci S. Effect of sphincter-sacrificing surgery for rectal carcinoma on quality of life in Muslim patients. Dis Colon Rectum. 2002; 45:1359-1366.

50. Rasmussen OO, Petersen IK, Christiansen J. Anorectal function following low anterior resection. Colorectal Dis. 2003; 5:258-261.

51. Fazio VW, Fletcher J, Montague D. Prospective study of the effect of resection of the rectum on male sexual function. World J Surg. 1980; 4:149-152.

52. Bryant CL, Lunniss PJ, Knowles CH, Thaha MA, Chan CL. Anterior resection syndrome. Lancet Oncol. 2012; 13:e403e408. https://doi.org/10.1016/S1470-2045(12)70236-X.

53. D'Ambrosio G, Paganini AM, Balla A, Quaresima S, Ursi P, Bruzzone P, Picchetto A, Mattei FI, Lezoche E. Quality of life in non-early rectal cancer treated by neoadjuvant radiochemotherapy and endoluminal loco-regional resection (ELRR) by transanal endoscopic microsurgery (TEM) versus laparoscopic total mesorectal excision. Surgical Endoscopy and Other Interventional Techniques. 2016; 30:504-511.

54. Valsdottir EB, Yarandi SS, Marks JH, Marks GJ. Quality of life and fecal incontinence after transanal endoscopic microsurgery for benign and malignan trectal lesions. Surg Endosc. 2014; 28:193-202. https://doi.org/10.1007/s00464013-3155-5.

55. Coco C, Rizzo G, Mattana C, Gambacorta MA, Verbo A, Barbaro B, Vecchio FM, Pafundi DP, Mastromarino MG, Valentini V. Transanal endoscopic microsurgery after neoadjuvant radiochemotherapy for locally advanced extraperitoneal rectal cancer: short-term morbidity and functional outcome. Surg Endosc. 2013; 27:2860-2867. https://doi.org/10.1007/s00464-013-2842-6. 
56. Yau T, Watkins D, Cunningham D, Barbachano Y, Chau I, Chong G. Longitudinal assessment of quality of life in rectal cancer patients with or without stomas following primary resection. Dis Colon Rectum. 2009; 52:669-677. https://doi.org/10.1007/DCR. 0b013e31819eb970.

57. Verseveld M, de Graaf EJ, Verhoef C, van Meerten E, Punt CJ, de Hingh IH, Nagtegaal ID, Nuyttens JJ, Marijnen CA, de Wilt JH; CARTS Study Group. Chemoradiation therapy for rectal cancer in the distal rectum followed by organsparing transanal endoscopic microsurgery (CARTS study). Br J Surg. 2015; 102:853-860.

58. Swellengrebel HA, Marijnen CA, Verwaal VJ, Vincent A, Heuff G, Gerhards MF, van Geloven AA, van Tets WF, Verheij M, Cats A. Toxicity and complications of preoperative chemoradiotherapy for locally advanced rectal cancer. Br J Surg. 2011; 98:418-426.
59. Smart CJ, Korsgen S, Hill J, Speake D, Levy B, Steward M, Geh JI, Robinson J, Sebag-Montefiore D, Bach SP. Multicentre study of short-course radiotherapy and transanal endoscopic microsurgery for early rectal cancer. Br J Surg. 2016; 103:1069-1075.

60. Garcia-Aguilar J, Renfro LA, Chow OS, Shi Q, Carrero XW, Lynn PB, Thomas CR Jr, Chan E, Cataldo PA, Marcet JE, Medich DS, Johnson CS, Oommen SC, et al. Organ preservation for clinical T2N0 distal rectal cancer using neoadjuvant chemoradiotherapy and local excision (ACOSOG Z6041): results of an open-label, single-arm, multi-institutional, phase 2 trial. Lancet Oncol. 2015; 16:1537-1546. 